\title{
EXISTENCE RESULTS FOR $\phi$-LAPLACIAN BOUNDARY VALUE PROBLEMS ON TIME SCALES
}

\author{
ALBERTO CABADA
}

Received 24 January 2006; Revised 31 May 2006; Accepted 1 June 2006

This paper is devoted to proving the existence of the extremal solutions of a $\phi$-Laplacian dynamic equation coupled with nonlinear boundary functional conditions that include as a particular case the Dirichlet and multipoint ones. We assume the existence of a pair of well-ordered lower and upper solutions.

Copyright (c) 2006 Alberto Cabada. This is an open access article distributed under the Creative Commons Attribution License, which permits unrestricted use, distribution, and reproduction in any medium, provided the original work is properly cited.

\section{Introduction}

The method of lower and upper solutions is a very well-known tool used in the theory of ordinary and partial differential equations. It was introduced by Picard [14] and allows us to ensure the existence of at least one solution of the considered problem lying between a lower solution $\alpha$ and an upper solution $\beta$, such that $\alpha \leq \beta$. Combining these kinds of techniques with the monotone iterative ones (see [13] and references therein), one can deduce the existence of extremal solutions lying between the lower and the upper ones.

In recent years these techniques have been applied to difference equations $[7,9,15]$. So, existence results of suitable boundary value problems are obtained and the differences and the similarities between the discrete and the continuous problems are pointed out. For instance, in second-order ordinary differential equations, the existence of $\alpha \leq \beta$, a pair of well-ordered lower and upper solutions of the periodic problem, ensures the existence of at least one solution remaining in $[\alpha, \beta]$. This result is true for the periodic discrete centered problem

$$
\Delta^{2} u_{k}=f\left(t, u_{k+1}\right), \quad k \in\{0,1, \ldots, N-1\}, \quad u(0)=u(N), \quad \Delta u(0)=\Delta u(N),
$$

but it is false for the noncentered ones [4].

It is important to consider both situations under the same formulation, that is, to study equations on time scales. One can see in [2] that, provided that $f$ is a continuous 
function, the second-order Dirichlet problem

$$
u^{\Delta \Delta}(t)=f\left(t, u^{\sigma}(t)\right), \quad t \in[a, b], \quad u(a)=A, \quad u\left(\sigma^{2}(b)\right)=B,
$$

has at least one solution lying between a pair of well-ordered lower and upper solutions. This study has been continued in [5] for $n$ th-order periodic boundary value problems, in [11] for antiperiodic dynamic equations, and in [1] for second-order dynamic equations with dependence of the nonlinear term on the first derivative.

This paper is devoted to the study of the $\phi$-Laplacian problem, which arises in the theory of radial solutions for the $p$-Laplacian equation $\left(\phi(x)=|x|^{p-2} x\right)$ on an annular domain (see [12] and references therein) and has been studied recently for differential equations (see, e.g., $[6,10]$ ) and also for difference equations $[4,8]$. It can be treated in the framework of second-order equations with discontinuities on the spacial variables [10].

First we study the existence results for the following boundary value problem:

$$
\begin{gathered}
-\left[\phi\left(u^{\Delta}(t)\right)\right]^{\Delta}=f\left(t, u^{\sigma}(t)\right), \quad t \in \mathbb{T}^{\kappa^{2}} \equiv[a, b], \\
B_{1}(u(a), u)=0, \\
B_{2}\left(u, u\left(\sigma^{2}(b)\right)\right)=0 .
\end{gathered}
$$

We assume that the following conditions are fulfilled:

$\left(\mathrm{H}_{1}\right) f: I \times \mathbb{R} \rightarrow \mathbb{R}$ is a continuous function;

$\left(\mathrm{H}_{2}\right) \phi: \mathbb{R} \rightarrow \mathbb{R}$ is continuous, strictly increasing, $\phi(0)=0$, and $\phi(\mathbb{R})=\mathbb{R} ;$

$\left(\mathrm{H}_{3}\right) B_{1}: \mathbb{R} \times C(\mathbb{T}) \rightarrow \mathbb{R}$ is a continuous function, nondecreasing in the second variable; $B_{2}: C(\mathbb{T}) \times \mathbb{R} \rightarrow \mathbb{R}$ is a continuous function, nonincreasing in the first variable.

Remark 1.1. Note that the assumption $\phi(0)=0$ is not a restriction. By redefining $\bar{\phi}(x)=$ $\phi(x)-\phi(0)$, the same problem is considered.

It is clear that, by defining $B_{1}(x, \eta)=x-c_{0}$ and $B_{2}(\xi, y)=y-c_{1}$, these functional conditions include as a particular case the Dirichlet conditions

$$
u(a)=c_{0}, \quad u\left(\sigma^{2}(b)\right)=c_{1} .
$$

The multipoint boundary value conditions are given by

$$
B_{1}(x, \eta)=-x+\sum_{i=1}^{n} a_{i} \eta\left(t_{i}\right), \quad B_{2}(\xi, y)=y-\sum_{j=1}^{m} b_{j} \xi\left(s_{j}\right),
$$

with $n, m \in \mathbb{N}, a_{i}, b_{j} \geq 0$ for all $i=1, \ldots, n$ and $j=1, \ldots, m, a<t_{1}<\cdots<t_{n} \leq \sigma^{2}(b)$, and $a \leq s_{1}<\cdots<s_{m}<\sigma^{2}(b)$.

Now, choosing two $\Delta$-measurable sets $J_{0}, J_{1} \subset \mathbb{T}$ and $l, r \in \mathbb{N}$ odd, it is possible to consider nonlinear boundary conditions as

$$
u(a)=\int_{J_{0}} u^{l}(t) \Delta t, \quad u\left(\sigma^{2}(b)\right)=\int_{J_{1}} u^{r}(t) \Delta t
$$


or

$$
u(a)=\max _{t \in J_{0}} u(t), \quad u\left(\sigma^{2}(b)\right)=\min _{t \in J_{1}} u(t)
$$

In Section 2 we prove the existence of at least one solution of problem (1.3)-(1.5) lying between a lower solution $\alpha$ and an upper solution $\beta$, such that $\alpha \leq \beta$. Section 3 is devoted to warrant the existence of extremal solutions of problem (1.3)-(1.4) coupled in this case with the nonfunctional boundary condition

$$
B_{2}\left(u(a), u\left(\sigma^{2}(b)\right)\right)=0 .
$$

The exposed results improve the ones given in [2] when $\phi$ is the identity and the Dirichlet conditions are considered. In this case the regularity of the lower and the upper solutions is weakened, here corners in the graphs are allowed. Moreover they cover the existence results given in [4] for difference equations.

Before defining the concept of lower and upper solutions, we introduce the following notations:

$$
\begin{aligned}
& u\left(t^{+}\right)= \begin{cases}\lim _{s \rightarrow t^{+}} u(s) & \text { if } t \text { is right-dense, } \\
u(t) & \text { if } t \text { is right-scattered, }\end{cases} \\
& u\left(t^{-}\right)= \begin{cases}\lim _{s \rightarrow t^{-}} u(s) & \text { if } t \text { is left-dense, } \\
u(\rho(t)) & \text { if } t \text { is left-scattered. }\end{cases}
\end{aligned}
$$

Definition 1.2. Let $n \geq 0$ be given and let $a=t_{0}<t_{1}<t_{2}<\cdots<t_{n}<t_{n+1}=\sigma(b)$ be fixed. $\alpha \in C(\mathbb{T})$ is said to be a lower solution of problem (1.3)-(1.4) if the following properties hold.

(1) $\alpha^{\Delta}$ is bounded on $\mathbb{T}^{\kappa} \backslash\left\{t_{1}, \ldots, t_{n}\right\}$.

(2) For all $i \in\{1, \ldots, n\}$, there are $\alpha^{\Delta}\left(t_{i}^{-}\right), \alpha^{\Delta}\left(t_{i}^{+}\right) \in \mathbb{R}$ satisfying the following inequality:

$$
\alpha^{\Delta}\left(t_{i}^{-}\right)<\alpha^{\Delta}\left(t_{i}^{+}\right)
$$

(3) For all $i=0,1, \ldots, n, \phi\left(\alpha^{\Delta}\right) \in C^{1}\left(t_{i}, t_{i+1}\right)$ and it satisfies

$$
\begin{gathered}
-\left[\phi\left(\alpha^{\Delta}(t)\right)\right]^{\Delta} \leq f\left(t, \alpha^{\sigma}(t)\right), \quad t \in\left(t_{i}, t_{i+1}\right), \\
B_{1}(\alpha(a), \alpha) \geq 0 \geq B_{2}\left(\alpha, \alpha\left(\sigma^{2}(b)\right)\right) .
\end{gathered}
$$

$\beta \in C(\mathbb{T})$ is an upper solution of problem (1.3)-(1.5) if the reversed inequalities hold for suitable points $a=s_{0}<s_{1}<s_{2}<\cdots<s_{n}<s_{n+1}=\sigma(b)$.

We look for solutions $u$ of problem (1.3)-(1.5) belonging to the set

$$
\left\{u \in C(\mathbb{T}): u \in C^{1}\left(\mathbb{T}^{\mathcal{\kappa}}\right): \phi\left(u^{\Delta}\right) \in C^{1}([a, b])\right\} .
$$

We define $[\alpha, \beta]=\{v \in C(\mathbb{T}): \alpha(t) \leq v(t) \leq \beta(t)$ for all $t \in \mathbb{T}\}$. 


\section{Existence of solutions}

In this section, provided that hypotheses $\left(\mathrm{H}_{1}\right)-\left(\mathrm{H}_{3}\right)$ are satisfied, we prove the existence of at least one solution in the sector $[\alpha, \beta]$ of the problem (1.3)-(1.5). First we construct a truncated problem as follows.

Define $p(t, x)=\max \{\alpha(t), \min \{x, \beta(t)\}\}$ for all $t \in \mathbb{T}$ and $x \in \mathbb{R}$. Thus, we consider the following modified problem:

$$
\begin{gathered}
-\left[\phi\left(u^{\Delta}(t)\right)\right]^{\Delta}=f\left(t, p\left(\sigma(t), u^{\sigma}(t)\right)\right), \quad t \in[a, b], \\
u(a)=B_{1}^{*}(u)=p\left(a, u(a)+B_{1}(u(a), u)\right), \\
u\left(\sigma^{2}(b)\right)=B_{2}^{*}(u)=p\left(\sigma^{2}(b), u\left(\sigma^{2}(b)\right)-B_{2}\left(u, u\left(\sigma^{2}(b)\right)\right)\right) .
\end{gathered}
$$

Now, we prove the following three results for problem (2.1)-(2.3).

Lemma 2.1. If $u$ is a solution of (2.1)-(2.3), then $u \in[\alpha, \beta]$.

Proof. We will only see that $\alpha(t) \leq u(t)$ for every $t \in \mathbb{T}$. The case $u(t) \leq \beta(t)$ for all $t \in \mathbb{T}$ follows in a similar way.

By definition of $B_{1}^{*}$ and $B_{2}^{*}$, using (2.2) and (2.3), we have that $\alpha(a) \leq u(a) \leq \beta(a)$ and $\alpha\left(\sigma^{2}(b)\right) \leq u\left(\sigma^{2}(b)\right) \leq \beta\left(\sigma^{2}(b)\right)$.

Now, let $s_{0} \in\left(a, \sigma^{2}(b)\right)$ such that

$$
\begin{gathered}
\alpha\left(s_{0}\right)-u\left(s_{0}\right)=\max _{t \in \mathbb{T}}\{(\alpha-u)(t)\}>0, \\
(\alpha-u)(t)<(\alpha-u)\left(s_{0}\right) \quad \forall t \in\left(s_{0}, \sigma^{2}(b)\right] .
\end{gathered}
$$

As a consequence,

$$
(\alpha-u)^{\Delta}\left(s_{0}^{-}\right) \geq 0 \geq(\alpha-u)^{\Delta}\left(s_{0}^{+}\right)
$$

which tells us that there exists $i_{0} \in\{0, \ldots, n\}$ such that $s_{0} \in\left(t_{i_{0}}, t_{i_{0}+1}\right)$.

In the case when $s_{0}$ is a right-dense point of $\mathbb{T}$, we have that $\alpha-u \geq 0$ on $\left[s_{0}, s_{1}\right] \subset$ $\left(t_{i_{0}}, t_{i_{0}+1}\right)$ for some suitable $s_{1}>s_{0}$. So, for all $t \in\left[s_{0}, \rho\left(s_{1}\right)\right]$, it is satisfied that

$$
-\left[\phi\left(u^{\Delta}(t)\right)\right]^{\Delta}=f\left(t, \alpha^{\sigma}(t)\right) \geq-\left[\phi\left(\alpha^{\Delta}(t)\right)\right]^{\Delta},
$$

and, integrating on $[s, t] \subset\left(s_{0}, \rho\left(s_{1}\right)\right]$, we arrive at

$$
\phi\left(u^{\Delta}(t)\right)-\phi\left(\alpha^{\Delta}(t)\right) \leq \phi\left(u^{\Delta}(s)\right)-\phi\left(\alpha^{\Delta}(s)\right) .
$$

So, passing to the limit in $s$, from the regularity of $\alpha$ and $u$ on $\left(t_{i_{0}}, t_{i_{0}+1}\right)$, we conclude that

$$
\phi\left(u^{\Delta}(t)\right)-\phi\left(\alpha^{\Delta}(t)\right) \leq \phi\left(u^{\Delta}\left(s_{0}^{+}\right)\right)-\phi\left(\alpha^{\Delta}\left(s_{0}^{+}\right)\right) \leq 0,
$$

for all $t \in\left(s_{0}, \rho\left(s_{1}\right)\right)$.

From this expression we arrive at $(\alpha-u)^{\Delta} \geq 0$ on $\left[s_{0}, \rho\left(s_{1}\right)\right]$, which contradicts the definition of $s_{0}$. 
When $s_{0}$ is right-scattered, we have, from (2.5), that

$$
(\alpha-u)^{\Delta}\left(s_{0}\right)<0
$$

If moreover $s_{0}$ is left-dense, the continuity of $(\alpha-u)^{\Delta}$ on $\left(t_{i_{0}}, t_{i_{0}+1}\right)$ implies that there exists an interval $V_{0} \subset\left(t_{i_{0}}, s_{0}\right)$ such that

$$
(\alpha-u)(t)>(\alpha-u)\left(s_{0}\right) \quad \forall t \in V_{0},
$$

which contradicts the definition of $s_{0}$.

Finally, when $s_{0}$ is isolated, we know that $(\alpha-u)^{\Delta}\left(\rho\left(s_{0}\right)\right) \geq 0>(\alpha-u)^{\Delta}\left(s_{0}\right)$ and

$$
-\left[\phi\left(u^{\Delta}\left(\rho\left(s_{0}\right)\right)\right)\right]^{\Delta}=f\left(\rho\left(s_{0}\right), \alpha\left(s_{0}\right)\right) \geq-\left[\phi\left(\alpha^{\Delta}\left(\rho\left(s_{0}\right)\right)\right)\right]^{\Delta} .
$$

Thus, we get at the following contradiction:

$$
\begin{aligned}
-\left[\phi\left(u^{\Delta}\left(s_{0}\right)\right)\right]+\left[\phi\left(u^{\Delta}\left(\rho\left(s_{0}\right)\right)\right)\right] & \geq-\left[\phi\left(\alpha^{\Delta}\left(s_{0}\right)\right)\right]+\left[\phi\left(\alpha^{\Delta}\left(\rho\left(s_{0}\right)\right)\right)\right] \\
& >-\left[\phi\left(u^{\Delta}\left(s_{0}\right)\right)\right]+\left[\phi\left(u^{\Delta}\left(\rho\left(s_{0}\right)\right)\right)\right] .
\end{aligned}
$$

LEMMA 2.2. If $u$ is a solution of problem (2.1)-(2.3), then $B_{1}(u(a), u)=0=B_{2}\left(u, u\left(\sigma^{2}(b)\right)\right)$. Proof. Suppose that $u\left(\sigma^{2}(b)\right)-B_{2}\left(u, u\left(\sigma^{2}(b)\right)\right)<\alpha\left(\sigma^{2}(b)\right)$. By definition of $B_{2}^{*}$, we obtain $u\left(\sigma^{2}(b)\right)=\alpha\left(\sigma^{2}(b)\right)$.

Thus, using the monotone properties of $B_{2}$ and Lemma 2.1, we conclude

$$
\alpha\left(\sigma^{2}(b)\right)>\alpha\left(\sigma^{2}(b)\right)-B_{2}\left(u, \alpha\left(\sigma^{2}(b)\right)\right) \geq \alpha\left(\sigma^{2}(b)\right)-B_{2}\left(\alpha, \alpha\left(\sigma^{2}(b)\right)\right) \geq \alpha\left(\sigma^{2}(b)\right),
$$

reaching a contradiction.

An analogous argument proves that $u\left(\sigma^{2}(b)\right)+B_{2}\left(u, u\left(\sigma^{2}(b)\right)\right) \leq \beta\left(\sigma^{2}(b)\right)$. In consequence, it is clear that condition (1.5) holds. In the same way we prove that (1.4) is verified.

Now we prove the existence of at least one solution of the modified problem.

Lemma 2.3. Let $\alpha$ and $\beta$ be a lower solution and an upper solution, respectively, for problem (1.3)-(1.5) such that $\alpha \leq \beta$ in $\mathbb{T}$. If hypotheses $\left(H_{1}\right)-\left(H_{3}\right)$ are satisfied, then problem (2.1)(2.3) has at least one solution.

Proof. Let $T: C(\mathbb{T}) \rightarrow C(\mathbb{T})$ be defined for all $t \in \mathbb{T}$ as

$$
T u(t)=B_{2}^{*}(u)-\int_{t}^{\sigma(b)} \phi^{-1}\left(\tau_{u}-\int_{a}^{r} f\left(s, p\left(\sigma(s), u^{\sigma}(s)\right)\right) \Delta s\right) \Delta r
$$

with $\tau_{u}$ the unique solution of the expression

$$
\int_{a}^{\sigma(b)} \phi^{-1}\left(\tau_{u}-\int_{a}^{r} f\left(s, p\left(\sigma(s), u^{\sigma}(s)\right)\right) \Delta s\right) \Delta r=B_{2}^{*}(u)-B_{1}^{*}(u) .
$$


It is not difficult to verify that $u$ is a fixed point of $T$ if and only if $u$ is a solution of (2.1)-(2.3).

First, we see that operator $T$ is well defined.

Let $u \in C(\mathbb{T})$ be fixed; we define the function $g_{u}: \mathbb{R} \rightarrow \mathbb{R}$ as follows:

$$
g_{u}(x)=\int_{a}^{\sigma(b)} \phi^{-1}\left(x-\int_{a}^{r} f\left(s, p\left(\sigma(s), u^{\sigma}(s)\right)\right) \Delta s\right) \Delta r \quad \forall x \in \mathbb{R} .
$$

Since $u$ is fixed, $g_{u}$ is a continuous and strictly increasing function on $\mathbb{R}$.

Note that the continuity of $f$ and the definition of $p$ imply that there exists $M>0$ independent of $u \in C(\mathbb{T})$ such that

$$
\left|f\left(t, p\left(\sigma(t), u^{\sigma}(t)\right)\right)\right| \leq M \quad \forall t \in \mathbb{T}^{\kappa} .
$$

Since $\phi^{-1}$ is increasing, we have, for each $x \in \mathbb{R}$, that

$$
\begin{aligned}
g_{-}(x) & \equiv(\sigma(b)-a) \phi^{-1}(x-(\sigma(b)-a) M) \leq g_{u}(x) \\
& \leq(\sigma(b)-a) \phi^{-1}(x+(\sigma(b)-a) M) \equiv g_{+}(x) .
\end{aligned}
$$

The functions $g_{ \pm}$are continuous, strictly increasing and, since $\phi(\mathbb{R})=\mathbb{R}, g_{ \pm}(\mathbb{R})=\mathbb{R}$. So, we have that $g_{u}(\mathbb{R})=\mathbb{R}$ for all $u \in C(\mathbb{T})$, and then for each $u \in C(\mathbb{T})$ there exists a unique $\tau_{u}$ satisfying $g_{u}\left(\tau_{u}\right)=B_{2}^{*}(u)-B_{1}^{*}(u)$ which is equivalent to the fact that $(2.16)$ is uniquely solvable for each $u \in C(\mathbb{T})$.

Now call $c(u)_{ \pm}=\left(g_{ \pm}\right)^{-1}\left(B_{2}^{*}(u)-B_{1}^{*}(u)\right)$. From (2.19) we deduce that

$$
c(u)_{+} \leq \tau_{u} \leq c(u)_{-} \quad \forall u \in C(\mathbb{T}) .
$$

And now, since $B_{2}^{*}(u)-B_{1}^{*}(u)$ is bounded in $C(\mathbb{T})$ and $\left(g_{ \pm}\right)^{-1}$ are continuous in $\mathbb{R}$, there exists $L>0$ such that

$$
\left|\tau_{u}\right| \leq L \quad \forall u \in C(\mathbb{T}) .
$$

Therefore (2.18) and (2.21) show that operator $T$ is bounded in $C(\mathbb{T})$.

Now, we prove that it is continuous.

Suppose $u_{n} \rightarrow u$ in $C(\mathbb{T})$. Let $\tau_{n}$ be related to $u_{n}$ by (2.16) and $\tau_{u}$ associated to $u$. Now we prove that $\lim _{n \rightarrow \infty} \tau_{n}=\tau_{u}$.

By construction of $\tau_{n}$ and $\tau_{u}$, we have

$$
\begin{aligned}
& B_{2}^{*}\left(u_{n}\right)-B_{1}^{*}\left(u_{n}\right)-B_{2}^{*}(u)+B_{1}^{*}(u) \\
& =\int_{a}^{\sigma(b)}\left[\phi^{-1}\left(\tau_{n}-\int_{a}^{r} f\left(s, p\left(\sigma(s), u_{n}^{\sigma}(s)\right)\right) \Delta s\right)-\phi^{-1}\left(\tau_{u}-\int_{a}^{r} f\left(s, p\left(\sigma(s), u^{\sigma}(s)\right)\right) \Delta s\right) \Delta r\right] .
\end{aligned}
$$

Thus, from the continuity of $p, B_{1}$, and $B_{2}$, we conclude that

$$
\lim _{n \rightarrow \infty} \int_{a}^{\sigma(b)} \phi^{-1}\left(\tau_{n}-\int_{a}^{r} f\left(s, p\left(\sigma(s), u_{n}^{\sigma}(s)\right)\right) \Delta s\right) \Delta t=\int_{a}^{\sigma(b)} \phi^{-1}\left(\tau_{u}-\int_{a}^{r} f\left(s, p\left(\sigma(s), u^{\sigma}(s)\right)\right) \Delta s\right) \Delta t .
$$


From the fact that $\left\{\tau_{n}\right\}$ is a bounded sequence in $\mathbb{R}$, we conclude that there exists a subsequence $\left\{\tau_{n_{k}}\right\}$ converging to a real number $\gamma=\lim \sup \left\{\tau_{n}\right\}$. Thus, from the continuity of $\phi^{-1}, p$, and $f$, we have

$\lim _{k \rightarrow \infty} \phi^{-1}\left(\tau_{n_{k}}-\int_{a}^{r} f\left(s, p\left(\sigma(s), u_{n_{k}}^{\sigma}(s)\right)\right) \Delta s\right)=\phi^{-1}\left(\gamma-\int_{a}^{r} f\left(s, p\left(\sigma(s), u^{\sigma}(s)\right)\right) \Delta s\right) \quad \forall r \in \mathbb{T}$,

and then

$$
\int_{a}^{\sigma(b)} \phi^{-1}\left(\tau_{u}-\int_{a}^{r} f\left(s, p\left(\sigma(s), u^{\sigma}(s)\right)\right) \Delta s\right) \Delta r=\int_{a}^{\sigma(b)} \phi^{-1}\left(\gamma-\int_{a}^{r} f\left(s, p\left(\sigma(s), u^{\sigma}(s)\right)\right) \Delta s\right) \Delta r .
$$

Since $\phi^{-1}$ is a strictly increasing function, we conclude that $\tau_{u}=\gamma$.

Analogously, we verify that $\tau_{u}=\liminf \left\{\tau_{n}\right\}$.

Now, since

$$
\begin{aligned}
& \left|\tau_{n}-\int_{a}^{t} f\left(s, p\left(\sigma(s), u_{n}^{\sigma}(s)\right)\right) \Delta s-\tau_{u}+\int_{a}^{t} f\left(s, p\left(\sigma(s), u^{\sigma}(s)\right)\right) \Delta s\right| \\
& \quad \leq\left|\tau_{n}-\tau_{u}\right|+\int_{a}^{\sigma(b)}\left|f\left(s, p\left(\sigma(s), u^{\sigma}(s)\right)\right)-f\left(s, p\left(\sigma(s), u_{n}^{\sigma}(s)\right)\right)\right| \Delta s \quad \forall t \in \mathbb{T},
\end{aligned}
$$

the convergence of the sequence

$$
\left\{\tau_{n}+\int_{a}^{t} f\left(s, p\left(\sigma(s), u_{n}^{\sigma}(s)\right)\right) \Delta s\right\}
$$

is uniform on $\mathbb{T}$.

Now, by using the uniform continuity of $\phi^{-1}$ on compact intervals, we conclude that

$$
T u_{n} \longrightarrow T u \quad \text { uniformly on } \mathbb{T} \text {. }
$$

Now we are going to prove that $T(C(\mathbb{T}))$ is a relatively compact set in $C(\mathbb{T})$.

Using (2.18), (2.21), and $\left(\mathrm{H}_{2}\right)$, we have that there exists $Q>0$ such that

$$
\phi^{-1}(-Q) \leq(T u)^{\Delta}(t) \leq \phi^{-1}(Q) \quad \forall t \in \mathbb{T}^{\mathcal{k}}, u \in C(\mathbb{T}) .
$$

As a consequence, the set $T(C(\mathbb{T}))$ is uniformly equicontinuous:

$$
|T u(t)-T u(s)|=\left|\int_{s}^{t}(T u)^{\Delta}(r) \Delta r\right| \leq \max \left\{\phi^{-1}(-Q), \phi^{-1}(Q)\right\}|t-s|,
$$

for all $s, t \in \mathbb{T}$.

Now, since $T(C(\mathbb{T}))$ is bounded, the Ascoli-Arzelá theorem [3, Theorem IV.24] ensures that operator $T$ is compact. Using the Tychonoff-Schauder fixed point theorem, see [2, Theorem 6.49], we know that there is at least one fixed point of $T$; hence a solution of (2.1)-(2.3). 
Now, we are in a position to enunciate the following existence result. The proof is a direct consequence of the three previous lemmas.

THEOREM 2.4. Let $\alpha$ and $\beta$ be a lower solution and an upper solution, respectively, for problem (1.3)-(1.5) such that $\alpha \leq \beta$ in $\mathbb{T}$. Assume that hypotheses $\left(H_{1}\right)-\left(H_{3}\right)$ are satisfied. Then problem (1.3)-(1.5) has at least one solution $u \in[\alpha, \beta]$.

\section{Existence of extremal solutions}

In this section we prove that the problem (1.3), (1.4), (1.10) has extremal solutions on $[\alpha, \beta]$, that is, the problem has a unique solution on $[\alpha, \beta]$ or there is a pair of solutions $v \leq w$ in $[\alpha, \beta]$ such that any other solution $u$ in that sector satisfies $v \leq u \leq w$.

THEOREM 3.1. Let $\alpha$ and $\beta$ be a lower solution and an upper solution, respectively, for problem (1.3), (1.4), (1.10) (with obvious notation) such that $\alpha \leq \beta$ in $\mathbb{T}$. Assume that hypotheses $\left(H_{1}\right)-\left(H_{3}\right)$ are satisfied. Then problem (1.3), (1.4), (1.10) has extremal solutions in $[\alpha, \beta]$.

Proof. Denote

$$
S:=\{v \in[\alpha, \beta]: v \text { is solution of }(1.3),(1.4),(1.10)\}
$$

As in the proof of Lemma 2.3, we can verify that the set

$$
S^{\Delta}:=\left\{v^{\Delta}: v \in S\right\}
$$

is bounded in the $C\left(\mathbb{T}^{\kappa}\right)$-norm.

So $S$ is closed, bounded, and uniformly equicontinuous. As a consequence, see [3, Theorem IV.24], we have that it is compact in $C(\mathbb{T})$.

Therefore, defining, for $t \in[a, b]$,

$$
v_{\min }(t):=\inf \{v(t): v \in S\}
$$

we have that, for each $t_{0} \in \mathbb{T}$, there is a function $v_{*} \in S$ such that

$$
v_{*}\left(t_{0}\right)=v_{\min }\left(t_{0}\right)
$$

and $v_{\min }$ is continuous in $\mathbb{T}$.

Now we prove that $v_{\min }$ is a solution of (1.3), (1.4), (1.10), showing that $v_{\min }$ is a limit of some sequence of elements of $S$, that is, for every $\varepsilon>0$, there exists $v \in S$ such that $\left\|v-v_{\min }\right\|_{C(\mathbb{T})} \leq \varepsilon$.

Fix $\varepsilon>0$ arbitrarily. As $S$ is an equicontinuous set and $v_{\min }$ is a continuous function, there exists $\mu>0$ such that for $t, s \in \mathbb{I}$ with $|t-s|<\mu$ we have

$$
|v(t)-v(s)|<\frac{\varepsilon}{2}, \quad \forall v \in S \cup\left\{v_{\min }\right\} .
$$


Now fix $0<r<\mu$ and define $\left\{\delta_{0}, \delta_{1}, \ldots, \delta_{m}\right\} \subset \mathbb{\mathbb { V }}$ such that $\delta_{0}=a, \delta_{m}=\sigma^{2}(b)$, and for $i=1, \ldots, m-1$,

$$
\delta_{i}= \begin{cases}\sigma\left(\delta_{i-1}\right) & \text { if } \sigma\left(\delta_{i-1}\right)>\delta_{i-1}+r \\ \max \left\{t \in \mathbb{T} \backslash\left\{\delta_{i-1}\right\}: t \leq \delta_{i-1}+r\right\} & \text { otherwise. }\end{cases}
$$

It is clear that

$$
\begin{gathered}
\delta_{i} \geq \delta_{i-2}+r \quad \forall i=2, \ldots, m \\
\delta_{i}=\sigma\left(\delta_{i-1}\right) \quad \text { or } \quad 0<\delta_{i}-\delta_{i-1} \leq r<\mu \quad \forall i=1, \ldots, m .
\end{gathered}
$$

Denote $\beta_{0}(t) \equiv v_{a}(t)$, where $v_{a}$ is a function of $S$ that satisfies $v_{a}(a)=v_{\min }(a)$, and for $i \in\{1, \ldots, m\}$ define

$$
\beta_{i}(t) \equiv \beta_{i-1}(t) \quad \text { if } \beta_{i-1}\left(\delta_{i}\right)=v_{\min }\left(\delta_{i}\right)
$$

Otherwise, consider $v_{i} \in S$ such that

$$
v_{i}\left(\delta_{i}\right)=v_{\min }\left(\delta_{i}\right)
$$

and define

$$
\begin{aligned}
s_{i} & :=\inf \left\{t \in\left[\delta_{i-1}, \delta_{i}\right] \cap \mathbb{T}: v_{i}(s)<\beta_{i-1}(s) \forall s \in\left[t, \delta_{i}\right] \cap \mathbb{T}\right\}, \\
s_{i+1} & :=\sup \left\{t \in\left[\delta_{i}, \sigma^{2}(b)\right] \cap \mathbb{T}: v_{i}(s)<\beta_{i-1}(s) \forall s \in\left[\delta_{i}, t\right] \cap \mathbb{T}\right\},
\end{aligned}
$$

and the function

$$
\beta_{i}(t)= \begin{cases}\beta_{i-1}(t) & \text { if } t \in\left(\left[a, s_{i}\right) \cup\left(s_{i+1}, \sigma^{2}(b)\right)\right] \cap \mathbb{T}, \\ v_{i}(t) & \text { if } t \in\left[s_{i}, s_{i+1}\right] \cap \mathbb{T} .\end{cases}
$$

Since function $\beta_{m}$ is a $C^{1}$ function except, at most, at the set

$$
A_{\beta}=\left\{s_{i}\right\}_{i=1}^{m+1} \cup\left\{\rho\left(s_{i}\right)\right\}_{i=1}^{m+1} \cup\left\{\sigma\left(s_{i}\right)\right\}_{i=1}^{m+1},
$$

it is clear that, by construction,

$$
\beta_{m}^{\Delta}\left(s^{-}\right) \geq \beta_{m}^{\Delta}\left(s^{+}\right) \quad \forall s \in A_{\beta},
$$

and coincides with a solution in $\left(\sigma\left(s_{i}\right), \rho\left(s_{i+1}\right)\right)$, we have that the regularity hypotheses in Definition 1.2 hold.

Now, from the definition of $\beta_{m}$ and $\left(\mathrm{H}_{3}\right)$, we have

$$
\begin{gathered}
B_{1}\left(\beta_{m}(a), \beta_{m}\right)=B_{1}\left(v_{a}(a), \beta_{m}\right) \leq B_{1}\left(v_{a}(a), v_{a}\right)=0, \\
B_{2}\left(\beta_{m}(a), \beta_{m}\left(\sigma^{2}(b)\right)\right)=B_{2}\left(\beta_{m}(a), v_{m}\left(\sigma^{2}(b)\right)\right) \geq B_{2}\left(v_{m}(a), v_{m}\left(\sigma^{2}(b)\right)\right)=0 .
\end{gathered}
$$


Thus, we have that $\beta_{m}$ is an upper solution of (1.3), (1.4), (1.10). By Theorem 2.4, there is a solution $w_{m}$ of $(1.3),(1.4),(1.10)$ such that $w_{m} \in\left[\alpha, \beta_{m}\right]$. So, by the construction of $\beta_{m}$,

$$
v_{\min }\left(\delta_{i}\right) \leq w_{m}\left(\delta_{i}\right) \leq \beta_{m}\left(\delta_{i}\right)=v_{\min }\left(\delta_{i}\right) \quad \forall i \in\{0, \ldots, m\}
$$

Now, let $t \in \mathbb{T} \backslash\left\{\delta_{0}, \ldots, \delta_{m}\right\}$. By construction, we know that there is $i \in\{1, \ldots, m\}$ such that $t \in\left(\delta_{i-1}, \delta_{i}\right)$ with $\delta_{i}-\delta_{i-1} \leq r$ (in other case $\delta_{i}=\sigma\left(\delta_{i-1}\right)$ and so $\left(\delta_{i-1}, \delta_{i}\right) \cap \mathbb{T}$ is empty).

As a consequence, by (3.5),

$$
\begin{aligned}
\left|w_{m}(t)-v_{\min }(t)\right| & \leq\left|w_{m}(t)-w\left(\delta_{i}\right)\right|+\left|w_{m}\left(\delta_{i}\right)-v_{\min }(t)\right| \\
& =\left|w_{m}(t)-w_{m}\left(\delta_{i}\right)\right|+\left|v_{\min }\left(\delta_{i}\right)-v_{\min }(t)\right|<\varepsilon .
\end{aligned}
$$

Then

$$
\left\|w_{m}-v_{\min }\right\|_{C(\mathbb{T})}<\varepsilon
$$

As $\varepsilon$ is arbitrary, by the compactness of $S$ on $C(\mathbb{T})$, we conclude that

$$
v_{\min } \in S
$$

Analogous arguments show us that problem (1.3), (1.4), (1.10) has a maximal solution $v_{\max } \in S$.

\section{References}

[1] F. M. Atici, A. Cabada, C. J. Chyan, and B. Kaymakçalan, Nagumo type existence results for secondorder nonlinear dynamic BVPs, Nonlinear Analysis 60 (2005), no. 2, 209-220.

[2] M. Bohner and A. Peterson, Dynamic Equations on Time Scales. An Introduction with Applications, Birkhäuser, Massachusetts, 2001.

[3] H. Brezis, Analyse fonctionnelle. Théorie et applications, Collection Mathématiques Appliquées pour la Maîtrise, Masson, Paris, 1983.

[4] A. Cabada, Extremal solutions for the difference $\phi$-Laplacian problem with nonlinear functional boundary conditions, Computers \& Mathematics with Applications 42 (2001), no. 3-5, 593601.

[5] ___ Extremal solutions and Green's functions of higher order periodic boundary value problems in time scales, Journal of Mathematical Analysis and Applications 290 (2004), no. 1, 35-54.

[6] A. Cabada, P. Habets, and R. L. Pouso, Optimal existence conditions for $\phi$-Laplacian equations with upper and lower solutions in the reversed order, Journal of Differential Equations 166 (2000), no. 2, 385-401.

[7] A. Cabada and V. Otero-Espinar, Optimal existence results for nth order periodic boundary value difference equations, Journal of Mathematical Analysis and Applications 247 (2000), no. 1, 6786.

[8] ___ Existence and comparison results for difference $\phi$-Laplacian boundary value problems with lower and upper solutions in reverse order, Journal of Mathematical Analysis and Applications 267 (2002), no. 2, 501-521.

[9] A. Cabada, V. Otero-Espinar, and R. L. Pouso, Existence and approximation of solutions for firstorder discontinuous difference equations with nonlinear global conditions in the presence of lower and upper solutions, Computers \& Mathematics with Applications 39 (2000), no. 1-2, 21-33. 
[10] A. Cabada and R. L. Pouso, Extremal solutions of strongly nonlinear discontinuous second-order equations with nonlinear functional boundary conditions, Nonlinear Analysis. Theory, Methods \& Applications 42 (2000), no. 8, 1377-1396.

[11] A. Cabada and D. R. Vivero, Existence and uniqueness of solutions of higher-order antiperiodic dynamic equations, Advances in Difference Equations 2004 (2004), no. 4, 291-310.

[12] H. Dang and S. F. Oppenheimer, Existence and uniqueness results for some nonlinear boundary value problems, Journal of Mathematical Analysis and Applications 198 (1996), no. 1, 35-48.

[13] G. S. Ladde, V. Lakshmikantham, and A. S. Vatsala, Monotone Iterative Techniques for Nonlinear Differential Equations, Monographs, Advanced Texts and Surveys in Pure and Applied Mathematics, vol. 27, Pitman, Massachusetts, 1985.

[14] E. Picard, Sur l'application des méthodes d'approximations successives a l'étude de certaines équations différentielles ordinaires, Journal de Mathématiques Pures et Appliquées 9 (1893), $217-$ 271.

[15] W. Zhuang, Y. Chen, and S. S. Cheng, Monotone methods for a discrete boundary problem, Computers \& Mathematics with Applications 32 (1996), no. 12, 41-49.

Alberto Cabada: Departamento de Análise Matemática, Facultade de Matemáticas,

Universidade de Santiago de Compostela, 15782 Santiago de Compostela,

Galicia, Spain

E-mail address: cabada@usc.es 